\title{
Local properties of local multiplicity distributions in hadronic Z decay *
}

\author{
S.V.Chekanov ${ }^{\dagger}$ with W.Kittel and W.J.Metzger \\ High Energy Physics Institute Nijmegen (HEFIN), University of Nijmegen/NIKHEF, \\ P.O. Box 9010, 6500 GL Nijmegen, The Netherlands
}

for the L3 Collaboration

Preliminary results on local multiplicity fluctuations in hadronic $\mathrm{Z}$ decays are presented. The data were obtained using the L3 detector at LEP. It is investigated to what extent Monte-Carlo models, which are tuned to reproduce global event-shape variables and single-particle inclusive distributions, can describe the local fluctuations measured by means of bunching parameters.

\section{INTRODUCTION}

The investigation of the evolution of the probabilities $P_{n}(\delta)$ of detecting $n$ particles in ever smaller sizes $\delta$ of phase-space windows (bins) provides detailed information on QCD multihadron production beyond single-particle densities, without trivial constraints from charge- and energymomentum conservation. A deviation of this distribution from that expected for purely independent particle production can be attributed to dynamical local multiplicity fluctuations.

The important quest behind such a study is the understanding of the origin of short-range correlations between final-state particles. As a consequence of these correlations, the normalized factorial moments (NFMs) $F_{q}(\delta)$ of the local multiplicity distribution $P_{n}(\delta)$ exhibit a power-like increase with decreasing $\delta$, namely $F_{q}(\delta) \propto \delta^{-\phi_{q}}$ [1], where $\phi_{q}$ are constants called intermittency indices. This phenomenon reflects the peculiarity of $P_{n}(\delta)$ to become broader with decreasing $\delta$. Since NFMs satisfy the scaling property $F_{q}(\lambda \delta)=\lambda^{-\phi_{q}} F_{q}(\delta)$, this is widely regarded as evidence that the correlations exhibit a selfsimilar underlying dynamics.

Local fluctuations in $\mathrm{e}^{+} \mathrm{e}^{-}$-processes have already been studied is several experiments [2]. The data do exhibit approximate power-like rise of the

\footnotetext{
* Presented at the XXVII Symposium on Multiparticle Dynamics, September 8-12, 1997 Frascati-Rome, Italy.

${ }^{\dagger}$ On leave from Institute of Physics, AS of Belarus, Skaryna av.70, Minsk 220072, Belarus.
}

NFMs with a saturation at small $\delta$. The conclusion has been reached that such a phenomenon is a consequence of the multi-jet structure of events, i.e., groups of particles with similar angles resulting in spikes of particles as seen in selected phasespace projections. Parton showers, fragmentation, resonance decays and Bose-Einstein interference can all contribute to these correlations. It has been found that for the statistics used at that time current Monte-Carlo models can, in general, describe the data, even without additional tuning.

Recently, it has been realized that the factorialmoment method poorly reflects the information content of local fluctuations, since the NFM of order $q$ contains a trivial contamination from lowerorder correlation functions (see reviews [3]). As a result, rather different event samples can exhibit a very similar behaviour of the NFMs. The fact that subtle details in the behaviour of $P_{n}(\delta)$ are missing, together with the small statistics used, may be the reason why different Monte-Carlo models can reasonably describe the local fluctuations measured in $\mathrm{e}^{+} \mathrm{e}^{-}$annihilation so far.

Another shortcoming of the factorial-moment measurement is that in moving to ever smaller phase-space bins, the statistical bias due to a finite event sample $\left(N_{\mathrm{ev}} \neq \infty\right)$ becomes significant, especially for high-order moments $q$. This is because in actual measurements the NFMs at small bin size are determined by the first few terms in the definition of the NFMs. In most cases this leads to a significant underestimate of the mea- 
Table 1

NFMs and BPs for the distributions quoted.

\begin{tabular}{lrrr}
\hline Distribution & \multicolumn{1}{c}{$P_{n}$} & NFMs & BPs \\
\hline Positive-binomial & $C_{n}^{N} p^{n}(1-p)^{N-n}$ & $\prod_{i=1}^{q}\left(1-\frac{i}{N}\right)$ & $\frac{q-1-N}{q-2-N}$ \\
Poisson & $p^{n} \exp (-p) / n !$ & 1 & 1 \\
Negative-binomial & $\frac{\Gamma(n+k)}{\Gamma(n+1) \Gamma(k)} p^{n}(p+1)^{-(n+k)}$ & $\prod_{i=1}^{q}\left(1+\frac{i}{k}\right)$ & $\frac{q-1+k}{q-2+k}$ \\
Geometric & $p^{n}(p+1)^{-n-1}$ & $\prod_{i=1}^{q}(1+i)$ & $\frac{q}{q-1}$ \\
\hline
\end{tabular}

sured NFMs with respect to their true values.

Cumulants are a more sensitive statistical tool (see [3] and references therein). However, their measurement is rather difficult and was rarely attempted. Besides, the cumulants are expected to be influenced by the statistical bias to even larger degree, since they are constructed from the factorial moments of different orders $q$.

\section{LOCAL PROPERTIES}

An important step towards an improvement of experimental measurements of the local multiplicity distribution was made in [4.5], where it was shown that any complex distribution can be represented as

$$
P_{n}(\delta)=P_{0}(\delta) \frac{\lambda^{n}}{n !} L_{n}, \quad L_{n}=\prod_{i=2}^{n} \eta_{i}^{n-i+1}(\delta),
$$

where $\lambda=P_{1}(\delta) / P_{0}(\delta)$. The factor $L_{n}$ measures a deviation of the distribution from a Poisson, for which $L_{n}=1$. Non-poissonian fluctuations, therefore, exhibit themselves as a deviation of $L_{n}$ from unity. The $L_{n}$ are constructed from the bunching parameters (BPs)

$\eta_{q}(\delta)=\frac{q}{q-1} \frac{P_{q}(\delta) P_{q-2}(\delta)}{P_{q-1}^{2}(\delta)}, \quad q>1$.

The expressions for the BPs and NFMs for some popular distributions are shown in Table 1. The most interesting observation is that while the NFM is an "integral" characteristic of the $P_{n}(\delta)$ and the BP is a "differential" one, both tools have values larger than unity if the distribution is broader than a Poisson. Generally, however, one should not expect that all BPs are larger than unity for a broad distribution; BPs probe the distribution locally, i.e. they are simply determined by the second-order derivative of the logarithm of $P_{n}(\delta)$ with respect to $n$. Note that in the case of local distributions, the width of the distribution is mainly determined by $\eta_{2}(\delta)$. This observation is based on the simple fact that $P_{n}(\delta)$ ceases to be bell-shaped at sufficiently small $\delta$.

BPs are more sensitive to the variation in the shape of $P_{n}(\delta)$ with decreasing $\delta$ than are NFMs [6]. In the case of intermittent fluctuations, one should expect $\eta_{2}(\delta) \propto \delta^{-d_{2}}$. For multifractal local fluctuations, the $\eta_{q}(\delta)$ are $\delta$-dependent functions for all $q \geq 3$, while for monofractal behaviour $\eta_{q}(\delta)=$ const for $q \geq 3$ 顿.

From an experimental point of view, the BPs have the following important advantages [5]:

1) They are less severely affected by the bias from finite statistics than the NFMs, since the $q$ th-order BP resolves only the behaviour of the multiplicity distribution near multiplicity $n=q-$ 1 ;

2) For the calculation of the BP of order $q$, one needs to know only the $q$-particle resolution of the detector, not any higher-order resolution.

In this paper, we present an experimental investigation of local fluctuations in the final-state hadron system produced in $Z^{0}$ decays at $\sqrt{s}=$ 91.2 GeV. The final-state charged hadrons have been recorded with the L3 detector during the 1994 LEP running period. The calculations are based on approximately 1.0M selected hadronic events. We compare the data with the JETSET 7.4 PS [7], ARIADNE 4.08 [8] and HERWIG 
5.9 [9] models. The models have been tuned to the L3 data [10].

\section{ANALYSIS}

1) Horizontal BPs:

In order to reduce the statistical error on the observed local quantities when analyzing experimental data, we use the bin-averaged BPs 《4,5]:

$\eta_{q}(M)=\frac{q}{q-1} \frac{\bar{N}_{q}(M) \bar{N}_{q-2}(M)}{\bar{N}_{q-1}^{2}(M)}$

where $\bar{N}_{q}(M)=\frac{1}{M} \sum_{m=1}^{M} N_{q}(m, \delta), N_{q}(m, \delta)=$ being the number of events having $q$ particles in bin $m$ and $M=\Delta / \delta$ is the total number of bins ( $\Delta$ represents the size of full phase-space volume). To be able to study non-flat distributions, as for rapidity, we have to carry out a transformation from the original phase-space variable to one in which the underlying density is approximately uniform, as suggested by Białas, Gadzinski and Ochs [11].

2) Generalized integral BPs:

To study the distribution for spikes, we will consider the generalized integral BPs [5] using the squared pairwise four-momentum difference $Q_{12}^{2}=-\left(p_{1}-p_{2}\right)^{2}$. In this variable, the definition of the BPs is given by

$\chi_{q}\left(Q^{2}\right)=\frac{q}{q-1} \frac{\Pi_{q}\left(Q^{2}\right) \Pi_{q-2}\left(Q^{2}\right)}{\Pi_{q-1}^{2}\left(Q^{2}\right)}$,

where $\Pi_{q}\left(Q^{2}\right)$ represents the number of events having $q$ spikes of size $Q^{2}$ in the phase-space of variable $Q_{12}^{2}$, irrespective of how many particles are inside each spike.

To define the spike size, we shall use the socalled Grassberger-Hentschel-Procaccia counting topology for which a many-particle hyper-tube is assigned a size $Q^{2}$ corresponding to the maximum of all pairwise distances (see Fig. 1). For a Poissonian production, the BPs (3) are equal to unity for all $q$.

\subsection{In the rapidity variable}

In order to study fluctuations inside jets, in most investigations the fluctuations have been measured in the rapidity $y$ defined with respect

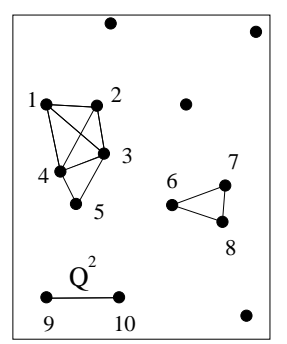

Figure 1. Example for the use of the GrassbergerHentschel-Procaccia counting topology in two dimensions. Full points illustrate the position of particles in an event. Points whose interparticle distance is smaller than or equal to the maximum $Q^{2}$, are connected by lines. This configuration corresponds to four spikes: one four-particle $(1,2,3,4)$, two three-particle $(3,4,5),(6,7,8)$ and one two-particle $(9,10)$ spikes.

to the thrust or sphericity axis [2]. The analysis for this variable is performed in the full rapidity range $|Y| \leq 5$. Fig. 2 shows the results for the BPs (2) for the rapidity variable after the Białas-Gazdzicki-Ochs transformation. The second-order BP decreases with increasing $M$ up to $M \simeq 20$, which is found to correspond to the value of $M$ at which the maximum of the multiplicity distribution $P_{n}(\delta)$ first occurs at $n=0$. At large $M$, all BPs show a power-law increase with increasing $M, \eta_{q} \sim M^{\alpha_{q}}$. This indicates that the fluctuations in $y$ defined with respect to the thrust axis are multifractal scale invariant. Note that the conclusion that fluctuations have a multifractal structure is possible without the necessity of calculating the intermittency indices $\phi_{q}$. In contrast, to reveal multifractality with the help of the NFMs, one first needs to carry out fits of the NFMs by a power law.

Both JETSET and ARIADNE agree well with the data for the higher orders. However, ARIADNE underestimates $\eta_{2}$ for all bin sizes while JETSET is significantly too low only for wide bins. HERWIG predictions (dashed lines) significantly overestimate the second-order BP obtained from the data. Since the second-order BP is determined by the dispersion of the distribution, this means that the HERWIG produces too broad local multiplicity distributions. Such a re- 


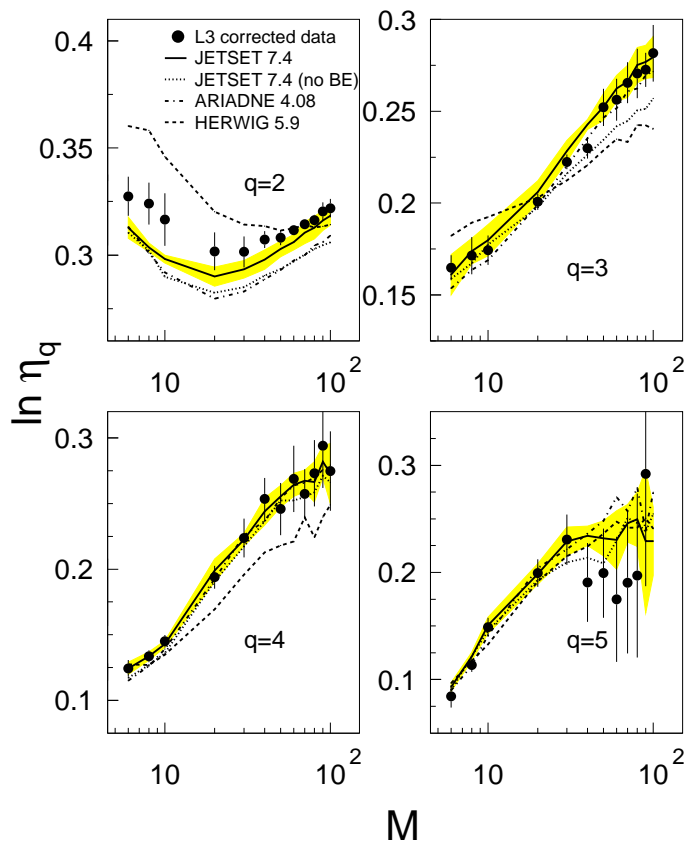

Figure 2. BPs as a function of the number $M$ of bins in rapidity defined with respect to the thrust axis. The shaded areas represent the statistical and systematic errors on the JETSET predictions.
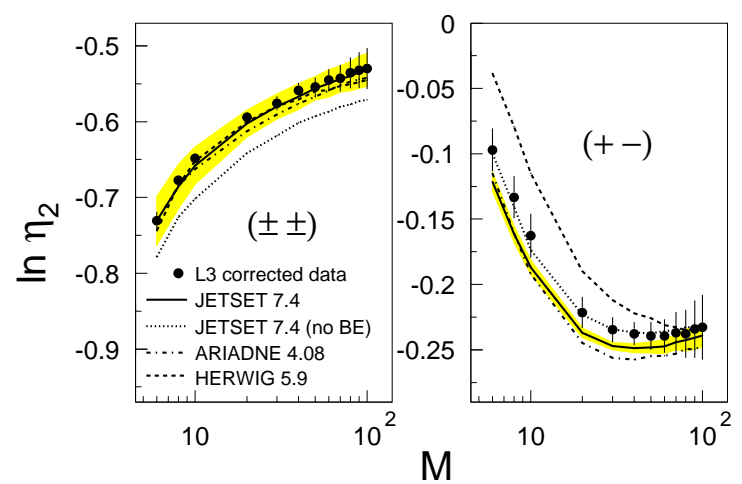

Figure 3. The second-order BP as a function of the number $M$ of bins in rapidity defined with respect to the thrust axis for like-charged and unlike-charged particle combinations. The shaded areas represent the errors on the JETSET predictions. sult confirms that obtained by the ALEPH Collaboration [12].

To study the disagreement in more detail, we split $\eta_{2}$ into two BPs:

$\eta_{2}=\eta_{2}^{( \pm \pm)}+\eta_{2}^{(+-)}$.

Here, $\eta_{2}^{( \pm \pm)}$is defined by (2) with $N_{2}(m, \delta)=$ $N_{2}^{( \pm \pm)}(m, \delta y)$, where $N_{2}^{( \pm \pm)}(m, \delta y)$ is the number of events having like-charged two-particle combinations inside bin $m$ of size $\delta y$. Analogously, $\eta_{2}^{(+-)}$is constructed from the number of events $N_{2}^{(+-)}(m, \delta y)$ having unlike-charged two-particle combinations. Note that due to a combinatorial reason, $\eta_{2}^{( \pm \pm)}<\eta_{2}^{(+-)}$.

Fig. 3 shows that $\eta_{2}^{( \pm \pm)}$and $\eta_{2}^{(+-)}$indeed behave completely differently. While $\eta_{2}^{( \pm \pm)}$has the expected rise, $\eta_{2}^{(+-)}$shows a strong decrease at low $M$ and an onset of increase only at large $M$. The structure of $\eta_{2}$ observed in Fig. 2 is a combination of these two effects.

Let us remind that, in order to model the $\mathrm{BE}$ interference in JETSET, the momenta of identical final-state particles are shifted to reproduce the expected two-particle correlation function. The main disadvantage of such an ad hoc method is that it spoils the overall energy-momentum conservation thus makes it necessary to modify also the momenta of non-identical particles to compensate for this. This effect can be seen in Fig. 3: JETSET without the BE correlations agrees with unlike-charged particles. However, JETSET with the BE effect disagrees with the data for unlikecharged particle combinations, while it agrees with like-charged.

The strong anti-bunching tendency seen for unlike-charged particles at $M<30$ can be attributed to resonance decays and to chain-like particle production along the thrust axis, as expected from the QCD-string model [13]. The latter effect leads to local charge conservation with an alternating charge structure. Evidence for this effect was recently observed by DELPHI [14]. As a result, there is a smaller rapidity separation between unlike-charged particles than between like-charged and $\eta_{2}^{(+-)}$is much larger than $\eta_{2}^{( \pm \pm)}$at small $M$. Having correlation lengths 
$\delta y \sim 0.5-1.0$ in rapidity, the resonance and the charge-ordering effects, however, become smaller with increasing $M$.

\subsection{In the four-momentum difference}

The study of BPs described above can help us to understand a tendency of the particles to be grouped into spikes inside small phase-space intervals. Another question is how the multiplicity of these spikes is distributed from event to event if the spike size goes to zero. To study this, we will use the BPs defined in (3).

Fig. 10 shows the behaviour of $\chi_{q}$ as a function of $-\ln Q^{2}$. The full lines represent the behaviour of the BPs in the Poissonian case. In contrast, all BPs obtained from the Monte Carlo models rise with increasing $-\ln Q^{2}$ (decreasing $Q^{2}$ ). This corresponds to a strong bunching effect of all orders, as expected for multifractal fluctuations. The anti-bunching effect $\left(\chi_{q}<1\right)$ for small $-\ln Q^{2}$ is caused by the energy-momentum conservation constraint [5].

To learn more about the mechanism of multiparticle fluctuations in the variable $Q_{12}^{2}$, we present in Fig. 5 the behaviour of the secondorder $\mathrm{BP}$ as a function of $-\ln Q^{2}$ for multiparticle hyper-tubes (spikes) made of like-charged and those of unlike-charged particles, separately. For this kind of observables, HERWIG gives better agreement than the LUND models.

\section{DISCUSSION}

Local multiplicity fluctuations were studied by means of bunching parameters. Since all highorder BPs show a power-like rise with decreasing size of phase-space interval, none of the conventional multiplicity distributions given in Table 1 can describe the observed local fluctuations.

For $\mathrm{e}^{+} \mathrm{e}^{-}$interactions, one can be confident that, at least on the parton level of this reaction, perturbative QCD can give a hint for the understanding of the problem. Analytical calculations based on the DLLA of perturbative QCD show that the multiplicity distribution of partons in ever smaller opening angles is inherently multifractal 15. Qualitatively, this is consistent with our results on the BPs for rapidity. Quan-
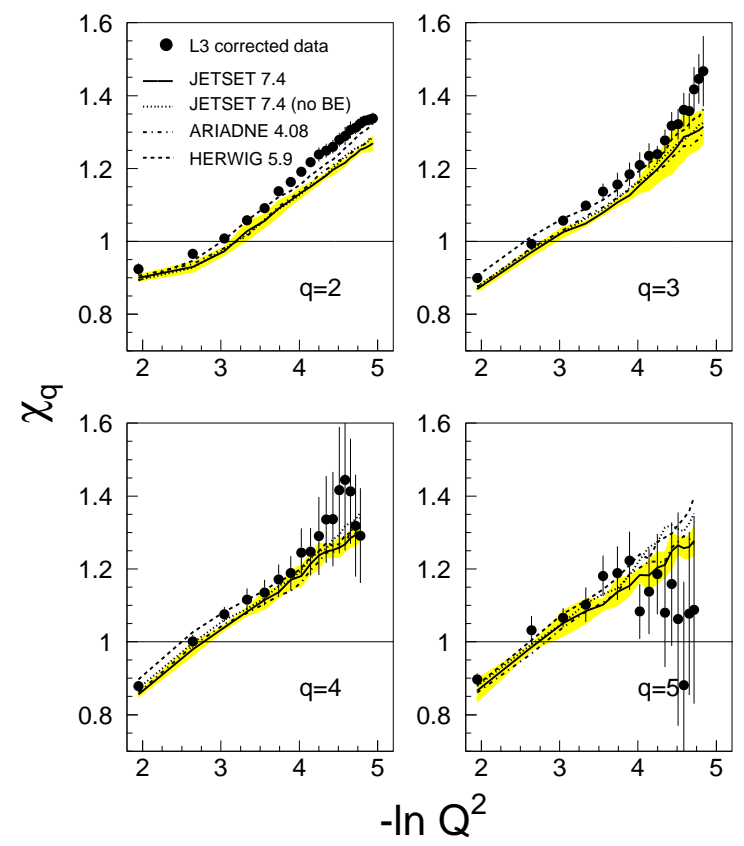

Figure 4. Generalized integral BPs as a function of the squared four-momentum difference $Q^{2}$ between two charged particles. The shaded areas represent the errors on the JETSET predictions.

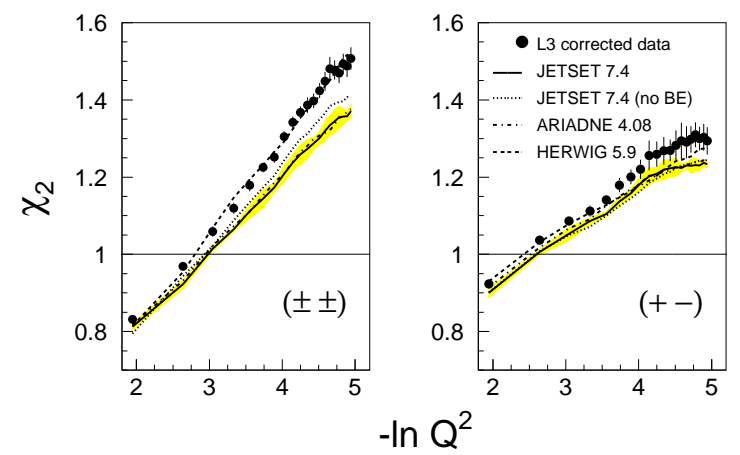

Figure 5. Generalized second-order BP as a function of the squared four-momentum difference $Q^{2}$ between two charged particles. The shaded areas represent the errors on the JETSET predictions. 
titatively, however, the QCD predictions disagree with the $\mathrm{e}^{+} \mathrm{e}^{-}$data and MC models [16].

In this paper we show that the power-law behaviour of BPs is mainly due to like-charged particles. JETSET gives the same power-law trend even without the BE effect. This means that the intermittency observed for like-charged particles appears to be largely a consequence of QCD parton showers and hadronization.

The predictions of the ARIADNE 4.08 model are comparable with those of the JETSET 7.4 PS model. This is essentially due to the same implementation of hadronization, which is based for both models on string fragmentation.

A noticeable disagreement is found between the data and HERWIG model for rapidity variable. The conversion of the partons into hadrons in LUND models is based on the Lund String Model 13. However, the hadronization in HERWIG is modelled with a cluster mechanism [9]. This difference can be a rather natural candidate to explain the observed disagreement. A particular concern is the large discrepancy for $\eta_{2}$. The behaviour of $\eta_{2}$ for not very small intervals is sensitive to low-multiplicity events, for which hadronization details could play a significant role.

\section{Acknowledgments}

This work is part of the research program of the "Stichting voor Fundamenteel Onderzoek der Materie (FOM)", which is financially supported by the "Nederlandse Organisatie voor Wetenschappelijk Onderzoek (NWO)". We acknowledge the effort of all engineers and technicians who have participated in the construction and maintenance of the LEP machine and the L3 detector.

\section{REFERENCES}

1. A.Białas and R.Peschanski, Nucl. Phys. B273 (1986) 703; Nucl. Phys. B308 (1988) 857.

2. TASSO Collab., W.Braunschweig et al., Phys. Lett. B231 (1989) 548 ; CELLO Collab., H.J.Behrend et al., Phys. Lett. B256 (1991) 97 ; OPAL Collab., M.Z.Akrawy et al., Phys. Lett. B262 (1991) 351 ; ALEPH Collab., D.Decamp et al., Z. Phys. C53
(1992) 21 ; DELPHI Collab., P.Abreu et al., Phys. Lett. B247 (1990) 137 ; DELPHI Collab., P.Abreu et al., Nucl. Phys. B386 (1992) 471 ; L3 Collab., B. Adeva et al., Z. Phys. C55 (1992) 39.

3. P.Bożek, M.Płoszajczak and R.Botet, Phys. Rep. 252 (1995) 101 ; E.A.De Wolf, I.M.Dremin and W.Kittel, Phys. Rep. 270 (1996) 1.

4. S.V.Chekanov and V.I.Kuvshinov, Acta Phys. Pol. B25 (1994) 1189 ; S.V.Chekanov, W.Kittel and V.I.Kuvshinov, Acta Phys. Pol. B27 (1996) 1739 ; S.V.Chekanov and V.I.Kuvshinov, J. Phys. G22 (1996) 601.

5. S.V.Chekanov, W.Kittel and V.I.Kuvshinov, Z. Phys. C73 (1997) 517.

6. S.V.Chekanov, V.I.Kuvshinov, J.Phys. G23 (1997) 951.

7. T.Sjöstrand, Comp. Phys. Comm. 82 (1994) 74.

8. L.Lönnblad, Comp. Phys. Comm. 71 (1992) 15.

9. G.Marchesini and B.R.Webber, Nucl. Phys. B310 (1988) 461 ; G.Marchesini et al., Comp. Phys. Comm. 67 (1992) 465.

10. J.Casaus, L3 Note 1946 (1996);

Sunanda Banerjee and Swagato Banerjee, L3 Note 1978 (1996) ; I.G.Knowles and T.Sjöstrand (conveners), "QCD Event Generators", Physics at LEP2, CERN-96-01, Vol. 2 (1996) p.103.

11. A.Białas and M.Gazdzicki, Phys. Lett. B252 (1990) 483 ; W.Ochs, Z. Phys. C50 (1991) 339 .

12. ALEPH Collab., D.Buskulic et al., Z. Phys. C69 (1995) 15.

13. B.Andersson, G.Gustafson, G.Ingelman, T.Sjöstrand, Phys. Rep. 97 (1983) 31.

14. DELPHI Collab., P.Abreu et al., Phys. Lett. B407 (1997) 174.

15. W.Ochs and J.Wosiek, Phys. Lett. B289 (1992) 159 ; B304 (1993) 144 ; Ph.Brax, J.L.Meunier and R.Peschanski, Z. Phys. C62 (1994) 649 ; Yu.Dokshitzer and I.M.Dremin, Nucl. Phys. B402 (1993) 139.

16. See the contributions of B.Buschbeck and W.Kittel to this symposium. 